\title{
Leben mit einer chronischen Krankheit
}

\author{
Martine Pilloud-Meyer
}

Dr. med., FMH in Psychiatrie-Psychotherapie

Auf einer schwindelerregenden Wanderung auf die Belle Etoile in den Savoyer Alpen im Sommer vor meinem 49. Geburtstag kam in mir die Frage auf: «Warum nur hat sich der Mensch aufgerichtet? Es ist so viel angenehmer auf allen vieren zu gehen!» «Mit dir stimmt etwas nicht!», kommentierte sogleich eine Stimme in meinem Inneren. Ich war tatsächlich kurz zuvor zweimal lang ausgestreckt in der Stadt auf dem Boden gelandet, ohne dass sich das geringste Hindernis auf meinem Weg befunden hätte. Dies gab mir zu denken: 14 Jahre zuvor hatte man bei mir eine Retrobulbärneuritis diagnostiziert. Ich hatte damals heftige Schmerzen im linken Auge, und alles, was ich ansah, hatte einen Braunstich. Für unser Staatsexamen hatten wir gelernt, dass bei einer Optikusnevritis weder der Arzt noch der Patient etwas sehen könne, ausserdem hatte niemand Schmerzen erwähnt ... So dachte ich nicht sofort an die richtige Diagnose. Unsere damals dreijährige Tochter, die keine Windeln mehr brauchte, nässte plötzlich wieder ihr Bett. Es war klar, dass ihre Mama sich grosse Sorgen machte. Man führte damals noch keine Magnetresonanz-Untersuchungen durch, und man empfahl mir eine Untersuchung mit evozierten Potentialen, das die vermutete Diagnose einer Entzündung des Sehnervs bestätigte. Es gab keine Möglichkeit, etwas zu unternehmen. Man konnte nur hoffen, dass mir die Krankheit vor ihrem wirklichen Ausbruch noch etwas Zeit lasse ...

Zu Beginn des Jahres, in dem ich meinen 50. Geburtstag feierte, konsultierte ich meinen Kollegen für Neurologie, mit dem ich eine angenehme, freundschaftliche Beziehung pflege. Er ist niedergelassen in der gleichen Kleinstadt, wo ich Psychiaterin bin. Ich teile viele Patienten mit ihm, der das Alter meines jüngsten Bruders hat und den ich seit dem Beginn meiner Praxistätigkeit vor 14 Jahren kenne. Ich bemerkte sofort sein Erschrecken, als ich mich als Patientin mit einer so belastenden Anamnese in seiner Praxis einfand. Ich fragte mich später, ob ich vielleicht einen mir weniger nahestehenden Kollegen hätte wählen sollen als behandelnden Neurologen, aber ich war immer befriedigt von seiner Arbeit. Ich spritzte mir während einer gewissen Zeit Interferon, hatte eine Pumpe mit Lioresal, zweifelte lange an deren Nützlichkeit, habe also vieles ausprobiert. Ernsthafte motorische Probleme sind etwa zehn Jahre später aufgetreten, aber glücklicherweise habe ich kaum kognitive Sorgen und funktioniere intellektuell befriedigend, obwohl ich inzwischen siebzig geworden bin.

Bei meiner Schwester, die elf Jahre jünger ist als ich, hat man um die zwanzig die gleiche Diagnose gestellt. Vor kurzem habe ich durch ein neu entdecktes Dokument erfahren, dass nicht nur meine Grossmutter väterlicherseits, sondern auch deren Mutter am Ende ihres Lebens an einer rätselhaften neurologischen Erkrankung litten, die sie daran hinderte, sich normal fortzubewegen! Die Diagnose einer multiplen Sklerose wurde natürlich nur bei meiner Schwester gestellt.

Ich habe am eigenen Leibe erfahren müssen, wie wenig Versicherungen chronische Krankheiten mögen, die auf alle möglichen Arten versuchen, sich von ihren Verpflichtungen zu befreien. Man kann sich kaum vorstellen, wie schwierig es ist, einen Rehabilitationsaufenthalt von drei Wochen in einer spezialisierten Klinik erlaubt zu bekommen! Dabei entlasten die regelmässigen Rehabilitationsaufenthalte die in die Pflege eingespannte Familie, erlauben eine einfache Bilanz des Gesundheitszustandes, geben neue Ideen für Verbesserungen, die oft auch Kosten einsparen! Aber selbst zwei physiotherapeutische Sitzungen pro Woche werden immer von neuem in Frage gestellt. Es ist sehr wichtig, trotz fortschreitender Erkrankung die physische Aktivität beizubehalten, und dazu braucht es regelmässige Physiotherapie. Ich fühle mich wie eine Betrügerin, gedemütigt durch das Verhalten der Versicherung. Ich muss ständig um die mir zustehenden Leistungen kämpfen. Ich bin Ärztin und im Besitz meines Intellekts - aber was tut jemand in schwächerer Position? Vielleicht habe ich so dank meiner Krankheit mehr Verständnis für die Sorgen meiner Patienten.

\section{Serie «Ärztinnen und Ärzte als Patienten»}

Haben Sie sich als Ärztin oder Arzt auch schon unversehens in der Patientenrolle wiedergefunden? Wie haben Sie diesen Perspektivenwechsel erlebt? Wenn Ihre Erfahrungen für die SÄZ-Leserschaft interessant sein könnten, möchten wir Sie dazu animieren, uns einen Artikel von maximal 8000 Zeichen Umfang an redaktion.saez[at]emh.ch einzureichen. Ihr Manuskript wird dann von der Redaktion geprüft und bei positiver Beurteilung im Rahmen der Serie "Ärztinnen und Ärzte als Patienten" publiziert. 